\title{
Microfluidic bacterial traps for simultaneous fluorescence and atomic force microscopy
}

\author{
Oliver Peric, Mélanie Hannebelle, Jonathan D. Adams ${ }^{\dagger}$, and Georg E. Fantner ( $\left.\varangle\right)$ \\ Laboratory for Bio- and Nano-Instrumentation, Institute of Bioengineering, School of Engineering, EPFL, 1015 Lausanne, Switzerland \\ ${ }^{\dagger}$ Present address: Department of Biosystems Science and Engineering, ETHZ, 4058 Basel, Switzerland
}

\author{
Received: 11 January 2017 \\ Revised: 18 March 2017 \\ Accepted: 27 March 2017 \\ (C) Tsinghua University Press \\ and Springer-Verlag Berlin \\ Heidelberg 2017

\section{KEYWORDS} \\ atomic force microscope \\ (AFM), \\ microfabrication, \\ microfluidics, \\ microbiology, \\ correlative microscopy, \\ physical immobilization
}

\begin{abstract}
The atomic force microscope has become an established research tool for imaging microorganisms with unprecedented resolution. However, its use in microbiology has been limited by the difficulty of proper bacterial immobilization. Here, we have developed a microfluidic device that solves the issue of bacterial immobilization for atomic force microscopy under physiological conditions. Our device is able to rapidly immobilize bacteria in well-defined positions and subsequently release the cells for quick sample exchange. The developed device also allows simultaneous fluorescence analysis to assess the bacterial viability during atomic force microscope imaging. We demonstrated the potential of our approach for the immobilization of rod-shaped Escherichia coli and Bacillus subtilis. Using our device, we observed buffer-dependent morphological changes of the bacterial envelope mediated by the antimicrobial peptide CM15. Our approach to bacterial immobilization makes sample preparation much simpler and more reliable, thereby accelerating atomic force microscopy studies at the single-cell level.
\end{abstract}

\section{Introduction}

Many bacterial cells have dimensions in the range of hundreds of nanometers to a few micrometers. This length scale is difficult to address with optical microscopy, and resolving details on the surface of living cells is nearly unattainable with optical or electron microscopy methods. The atomic force microscope (AFM), in contrast, is inherently well-suited for the nanoscale characterization of living cells. The analysis of the bacterial surface with nanometer precision was achieved on a routine basis over two decades ago with electron microscopy techniques [1]. The main drawback was that bacteria had to be prepared through special protocols that resulted in the death of the bacteria prior to imaging; thus, the analysis of dynamic changes of the bacterial envelope was not possible.

AFM analysis in a liquid medium allows the study of live microorganisms with nanometer precision. Pioneering AFM images of mammalian cells in physiological buffers [2] and the isolated bacterial envelope in liquid $[3,4]$ showed the great potential of

Address correspondence to georg.fantner@epfl.ch 
AFM analysis in a liquid medium for microbiology [5]. More recent findings have elucidated the mechanical properties of type IV pili, which have been found to play a key role in bacterial attachment to biotic and abiotic surfaces [6]. Furthermore, the peptidoglycan architecture of Bacillus subtilis bacteria was determined to be a coiled-coil model [7], and observations on genetically identical Escherichia coli bacteria showed that they reacted very differently at the nanoscale when treated with the same antibiotic [8]. The results obtained using AFM complement findings by traditional methods and expand our knowledge of biological systems $[9,10]$.

Nevertheless, the broad use of AFM in microbiology has in part been limited by the difficulty of sample preparation. Particularly for the observation of living bacteria under physiological conditions, the immobilization of bacteria on the surface is challenging [11, 12]. Various immobilization techniques have been developed over the years and can be classified into two distinct categories: chemical substrate modification [13] or the physical immobilization of bacteria [14].
For the chemical treatment of the substrate, typically a mica or glass surface, coating of the surface with poly-L-lysine is commonly used alongside coating with polyethylenimine or gelatin. The substrate surface is functionalized with positive charges, leading to the immobilization of the negatively charged bacterial envelope [15]. Covalent binding of the bacterial envelope to the substrate can be achieved by functionalizing a surface with amine groups, carboxyl groups, or glutaraldehyde [16]. Nevertheless, substrate modification methods often perform poorly in physiological buffers or compromise the bacterial viability $[16,17]$. Bacteria often fail to adhere strongly enough to the substrate [18] and higher forces applied by the AFM tip can detach a bacterium [19], as depicted in Figs. 1(a) and S1 in the Electronic Supplementary Material (ESM).

The physical immobilization of bacteria is therefore the most reliable method [20]. Pioneering results were achieved using Millipore ${ }^{\mathrm{TM}}$ filters, enabling the study of yeast cells [14] and coccoid bacteria [21]. These passive physical immobilization methods are highly suitable for round-shaped microorganisms, but cannot (a)

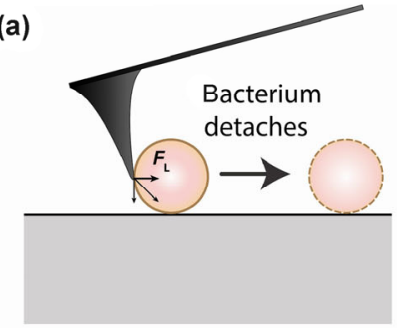

(c)

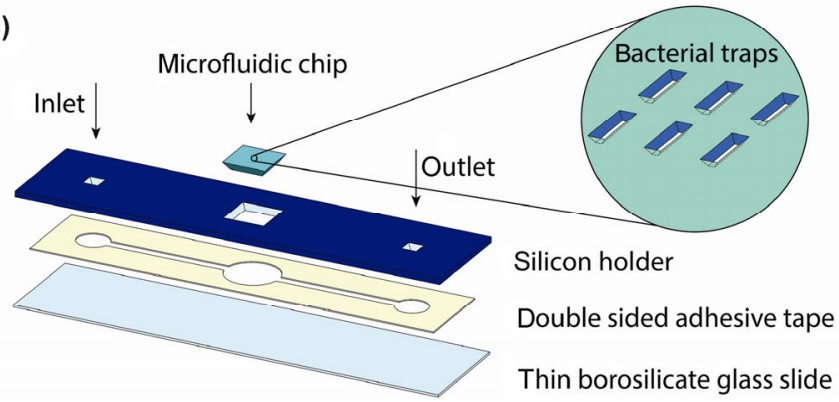

(b)

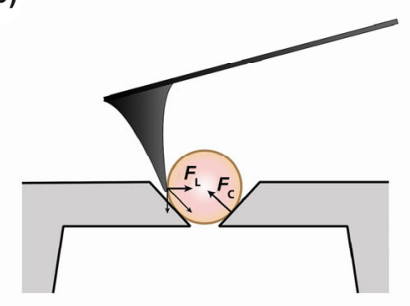

(d)

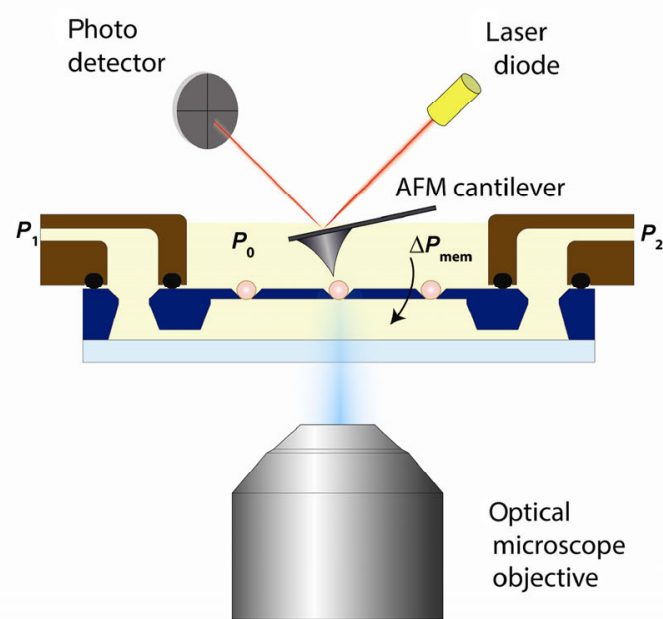

Figure 1 (a) Schematic of atomic force microscope (AFM) imaging of a bacterium. The lateral force $F_{\mathrm{L}}$ exerted by the AFM cantilever tip during scanning causes the bacterium to detach. (b) Proposed solution to immobilize bacteria for AFM imaging in traps with slanted sidewalls. The slanted walls of the trap exert a force $F_{\mathrm{C}}$, which counteracts the lateral forces $F_{\mathrm{L}}$ during the interaction of the bacterium with the sidewalls of the AFM cantilever tip. (c) Assembly of the microfluidic chip containing the bacterial traps. The microfluidic chip is mounted to the silicon holder in a central, square opening. The silicon holder is attached to a borosilicate glass slide through a prepatterned double-sided adhesive tape to form the microfluidic channels. (d) Measurement configuration for bacterial trapping in combined AFM/optical microscopy setup. A pressure difference $\Delta P_{\text {mem }}$ is applied across the membrane containing the bacterial traps. The top side is exposed to atmospheric pressure $P_{0}$, whereas the pressure in the channel is controlled through the input $P_{1}$ and output $P_{2}$. 
be reliably used to immobilize microbes of other shapes such as rod-shaped or filamentous bacteria.

A strong, robust immobilization method compatible with imaging under physiological conditions is still lacking, especially for high-speed AFM and force measurements of the bacterial envelope. In addition, reversible trapping at well-defined positions is favorable for experimental repeatability, without the need for tedious sample exchange and preparation. Correlative AFM/optical microscopy imaging is of particular interest, to correlate the surface topography with specific intracellular components, thereby making use of the many fluorescence probes available in microbiology. For such correlative experiments, the bacterial traps should be transparent and compatible with combined AFM/optical microscopy.

To fulfill these requirements and combine the benefits of the current physical immobilization techniques, we propose a microfluidic device with active immobilization. Bacteria are physically immobilized in V-shaped traps, where the lateral forces of the AFM tip during scanning are counteracted by the slanted walls (Fig. 1(b)). To guide bacteria towards the traps, we use pressure-driven flow, allowing the trapping and releasing of bacteria on demand. To be fully compatible with optical microscopy, the underside of the device is transparent. The microfluidic device thereby allows fluorescence microscopy from the bottom and AFM imaging in liquid from the top, as depicted schematically in Fig. 1(c).

\section{Experimental}

\subsection{Fabrication and characterization}

We fabricated V-shaped pits on a freestanding silicon membrane. The pits had apertures that were approximately $200 \mathrm{~nm}$ wide at the bottom, thus making a fluidic connection to the chamber underneath the membrane. The chamber was connected via microfluidic channels to the inlet and outlet ports, as shown in Fig. 1(d). By creating a pressure difference across the membrane, bacteria could be attracted to and subsequently trapped in the pits. By reversing the pressure across the membrane, trapped bacteria could be expelled from the pits for a rapid sample exchange.
Owing to the small size of bacteria, silicon-based microfabrication technology is most suitable for making the traps. For the primary microfluidic part, we used a silicon-on-insulator (SOI) wafer with a silicon device layer thickness of $340 \mathrm{~nm}$, which is marginally smaller than the radii of the rod-shaped bacteria, E. coli and $B$. subtilis. The process flow containing the main steps of the microfluidic chip fabrication is depicted in Fig. 2. We used silicon nitride as the etch mask for anisotropic wet-etching with potassium hydroxide $(\mathrm{KOH})$. The top side of the SOI wafer with the device layer was patterned by electron beam (e-beam) lithography, whereas the back side was patterned using standard photolithography. We relied on the anisotropic etch properties of $\mathrm{KOH}$ to create slanted walls in the silicon layer, and the silicon dioxide layer of the SOI was used as a $\mathrm{KOH}$ etch stop. After the first $\mathrm{KOH}$ etching, the V-shaped pits were protected

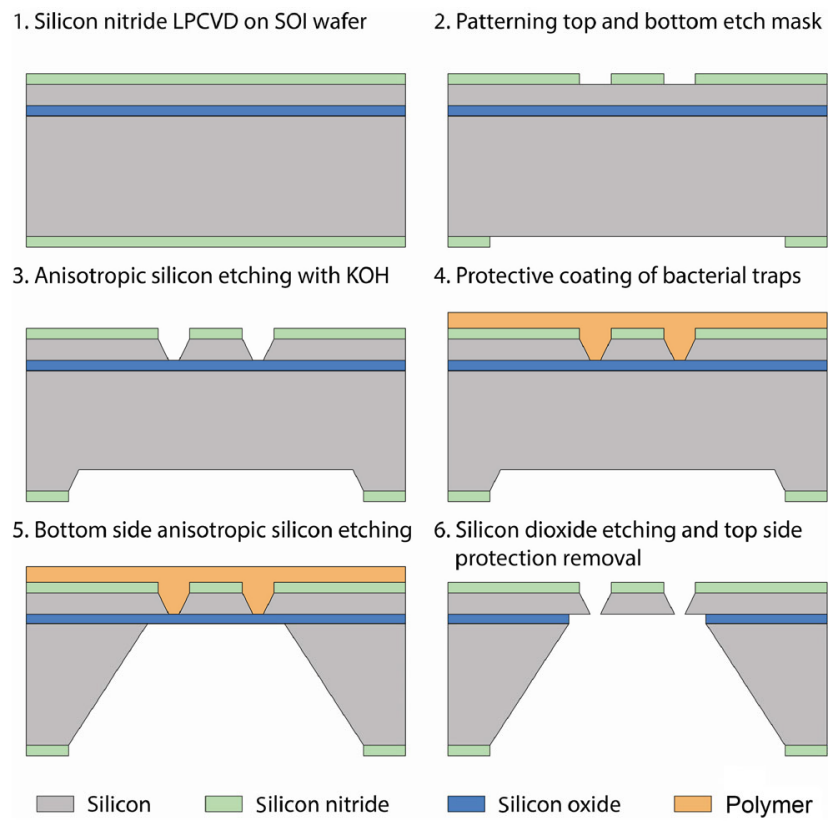

Figure 2 Main steps of the microfluidic chip process flow containing bacterial traps. (1) Low-pressure chemical vapor deposition of silicon nitride on a silicon-on-insulator wafer as the etch mask material. (2) Electron beam patterning on the device layer side and photolithography patterning on the back side of the wafer. (3) Fabrication of the bacterial traps through the first anisotropic wet-etching of silicon with $\mathrm{KOH}$. (4) Protection of the bacterial traps by spin-coating the top side of the wafer with a protective polymer layer. (5) Creation of a fluidic chamber by anisotropic wet-etching of the silicon on the back side of the wafer with $\mathrm{KOH}$. (6) Removal of silicon dioxide and the protective polymer coating using hydrofluoric acid. 
with a polymer coating (ProTEK ${ }^{\circledR}$ B3, Brewer Science, Rolla, MO, USA) and a consecutive $\mathrm{KOH}$ etch step was used to create the cavity underneath each trap. The protective polymer layer acts as a mechanical support for the microfluidic chips after the second $\mathrm{KOH}$ etch. The last processing step, consisting of silicon dioxide etching and removal of the polymer layer, was done on a chip-by-chip basis with hydrofluoric acid. Figures 3(a) and 3(b) depict bacterial traps at the end of the microfabrication process.

To increase the number of trapping devices obtainable from one SOI wafer, the bacterial traps were fabricated on small chips and assembled into chip holders made of conventional silicon wafers. For compatibility with optical microscopy, we assembled the chip holder with a borosilicate glass slide with high transmission and low autofluorescence values. The assembly of the microfluidic device is shown in Fig. 1(c). The assembly was placed between an AFM and an inverted optical microscope [22]. Bacteria in a liquid medium were placed on top of the fluidic chip,

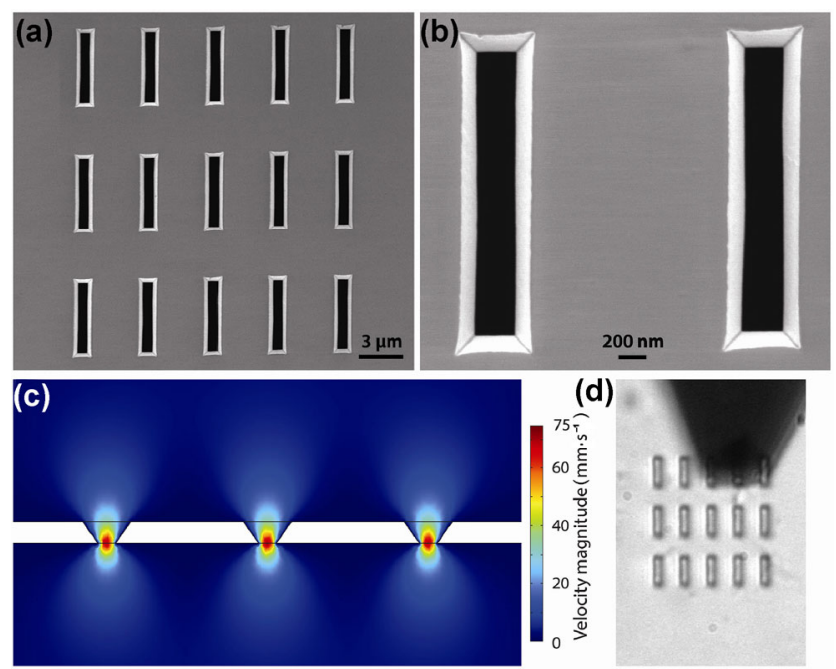

Figure 3 (a) Scanning electron microscope image of the array of bacterial traps after fabrication. The dimensions of each trap matched the size of an E. coli bacterium. (b) Close-up scanning electron microscope image of two traps. (c) Simulation of the flow velocity around the traps in a cross-section of the membrane. The pulling pressure applied below the membrane was -20 mbar, to match the experimental conditions. (d) Brightfield image from the inverted microscope showing the bacterial trap array with an engaged AFM cantilever during scanning. The image is taken with a $60 \times$ air objective lens through the borosilicate glass slide and the water-filled channel below the membrane. while the fluidic channel underneath was flushed with the same medium. By creating a pressure difference across the membrane containing bacterial traps, we could guide bacteria towards the traps or release them.

We used the protective polymer layer as a mechanical stabilization layer during the second $\mathrm{KOH}$ etch and consecutive cleaning steps. We were thus able to design the chip layout without any silicon support structures to the wafer, increasing the overall number of devices per wafer. Furthermore, the square design of the SOI chip allowed for easy integration into the silicon holder.

\subsection{Fluid flow analysis}

Using finite element analysis, we simulated the flow around the traps; see Fig. 3(c). The maximum velocity for water passing through the bacterial traps is $75 \mathrm{~mm} \cdot \mathrm{s}^{-1}$ for an applied pressure across the membrane of -20 mbar, as used during experiments. This translates to a volume flow between $3.0 \times 10^{-4}$ and $7.6 \times 10^{-4} \mu \mathrm{L} \cdot \mathrm{s}^{-1}$ for the trap array designs used during the experiments. Our estimate of the force acting on a trapped bacterium due to the applied pressure difference was approximately $6 \mathrm{nN}$, which is in the order of magnitude for lateral AFM forces exerted on a bacterium during scanning [19]. The pressure difference across the membrane was only necessary for the trapping process and could be completely switched off during AFM analysis. A bacterium did not need to be kept immobilized by the applied pressure difference, since the slanted walls of the bacterial traps counteracted the lateral forces exerted by the AFM tip during scanning, which is an empowering feature of the proposed immobilization technique. More details regarding the fluid flow analysis are given in the materials and methods section.

\section{Results and discussion}

By creating a pressure difference across the nanofluidic traps, we were able to attract bacteria towards the traps and physically immobilize them for AFM analysis. The bacterial traps were optically visible with the inverted microscope, as shown in Fig. 3(d), enabling fluorescence microscopy. The thickness of the 
microfluidic chip assembly allows the use of $60 \times$ and $100 \times$ long working distance objectives. Bacteria were trapped and subsequently imaged in lysogeny broth (LB) growth medium, using an applied pressure difference across the membrane of the microfluidic chip as low as 20 mbar. Figures 4(a) and S2(a) in the ESM show an immobilized non-motile E. coli bacterium, and Fig. S2(b) in the ESM shows the surface of an immobilized motile B. subtilis bacterium. Figure 4(b) shows the deformation map of an E. coli bacterium, overlaid with the height information represented in three dimensions (3D). As expected, the bacterium was more deformable than the surrounding trapping device. Through established theories [23-25] it is therefore possible to approximate the elastic modulus of the bacterium when the AFM cantilever tip radius is additionally determined [26].

During an experiment, particles smaller than the bottom opening of the nanofluidic traps could pass through, whereas particles larger or in the size of the targeted bacteria were trapped. By simply reversing the pressure difference, we could repel any undesirable particles from the traps and restart the immobilization of bacteria. Possible contaminants at the surface and in proximity of the bacterial traps could be eliminated by pipetting the fluid around the traps on the top side of the microfluidic chip. Moreover, by applying higher scanning forces with the AFM tip, anything (a)

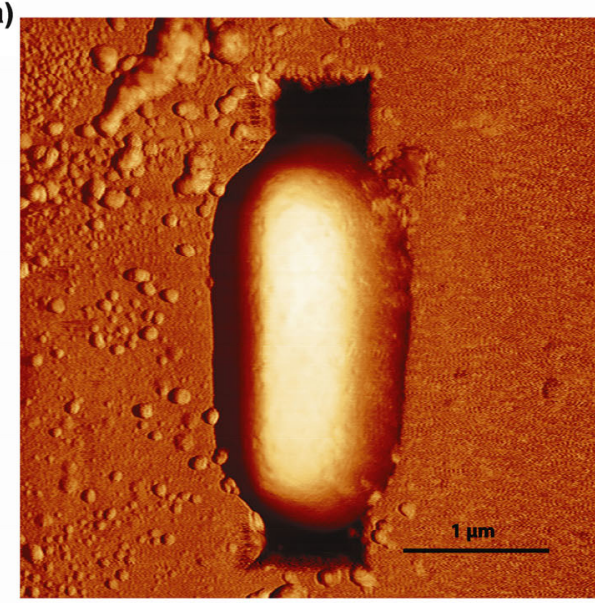

ए으

(b)

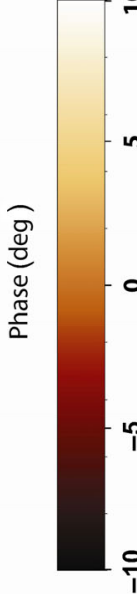

i

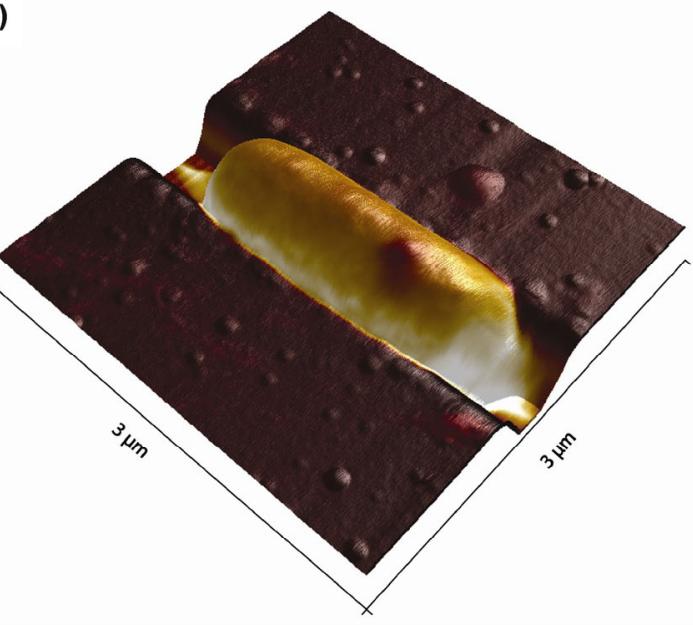

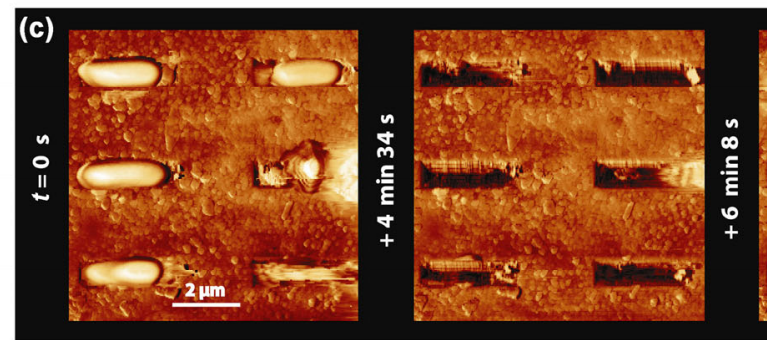

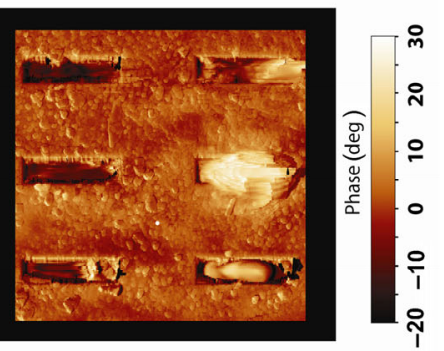

Pressure $(\mathrm{kPa})$

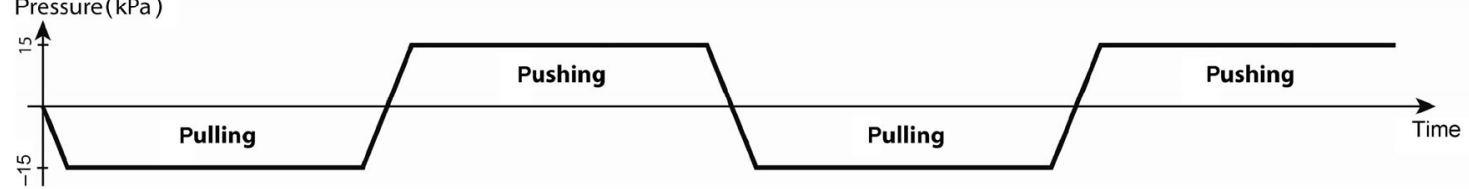

Figure 4 (a) AFM image of a trapped E. coli bacterium in lysogeny broth growth medium. The image was obtained in tapping mode at $2 \mathrm{~Hz}$ with a resolution of $1,024 \times 1,024$ pixels. (b) Three-dimensional representation of the height of an $E$. coli bacterium with color-coded deformation, where a brighter pixel value represents a higher indentation of the sample. The bacterium was analyzed in Milli- $Q^{\circledR}$ water in PeakForce $\mathrm{QNM}^{\mathbb{B}}$ mode (Bruker) with a resolution of $2,048 \times 2,048$ pixels. (c) Trapping and releasing process of bacteria during high-speed AFM imaging in tapping mode. The AFM phase images shown here were recorded with a resolution of $1,024 \times 256$ pixels and 10 lines $\cdot \mathrm{s}^{-1}$. For the trapping and releasing of bacteria, we created a pressure difference across the traps by controlling the pressure in the fluidic chamber underneath the traps. The applied pressure difference was \pm 150 mbar. 
not fully immobilized in the traps was swept away. Eventual clogging of the traps may be solved by the selective delivery of bacteria towards the traps or the use of a fluidic filter to distinguish between bacteria and particles of a certain size, as described by previous studies [27, 28]. However, bacteria can also squeeze through the traps if the pressure difference across the membrane is at least an order of magnitude higher than for the discussed experiments.

The fabricated bacterial traps can not only be used for spatially-defined bacterial trapping, but also allow the release of the immobilized bacteria on demand. Simply by inverting the pressure difference across the membrane, the immobilization mechanism is reversed and bacteria are released. The trapping and releasing processes are depicted in Fig. 4(c). We used a pressure of -150 mbar to immobilize the bacteria and a pressure of 150 mbar to release them from the traps. Switching of the pressure difference was performed during AFM imaging and the process was repeatable.

AFM imaging of bacteria has become a powerful tool for studying the interaction of antimicrobial agents with bacteria $[29,30]$. Pioneering results have shown that the $\beta$-lactam antibiotic cefodizime caused morphological changes of the E. coli envelope and could lead to lysis, depending on the concentration [31]. Staphylococcus aureus bacteria exposed to the peptidoglycan cleaving enzyme lysostaphin also showed a roughening of the bacterial surface and differences in the nanomechanical properties of the bacterial envelope [32].

Recent advances have shown that despite the rising resistance of pathogens to conventional antibiotics, antimicrobial peptides still exhibit potent bacterial killing [33-35]. Analogous to previous experiments [8], we exposed E. coli bacteria to the antimicrobial peptide CM15 and imaged the bacterial response over time. Since the immobilization efficiency of the traps is independent of the medium, we performed the experiment in both Milli-Q ${ }^{\circledR}$ water and physiological growth solution. We observed a medium-dependent response of the bacterial surface in the presence of CM15. While imaging a bacterium in Milli- $\mathrm{Q}^{\circledR}$ water, the bacterial surface roughened after the injection of the minimum inhibitory concentration (MIC) of CM15, as shown in Fig. S3 (in the ESM). In contrast, when the bacterium was imaged in LB growth medium, the injection of equal concentration of CM15 resulted in only a minimal increase in surface roughness (see Figs. 5(a) and 5(b)). Furthermore, the bacterial envelope regained its initial smooth surface within minutes. After an injection of five times the MIC of CM15, the bacterial envelope showed pronounced surface features that persisted until the end of the experiment.

These observations could indicate the presence of a bacterial envelope repair mechanism that is present and active under physiological conditions. It has been shown that osmoprotectants present in LB growth medium protect bacteria against the killing mediated by CM15 [36]. Our observations support the cytotoxicity of CM15 against E. coli and the reported findings regarding the influence of osmoprotectants on bacterial killing. Using bacterial viability stains, we were able to monitor the viability of the immobilized bacteria during simultaneous AFM analysis with the LIVE/ $\mathrm{DEAD}^{\circledR}$ BacLight $^{\mathrm{TM}}$ bacterial viability kit (Thermo Fisher Scientific, Waltham, MA, USA). Before the injection of CM15, the trapped bacteria were visible only in the green fluorescence channel, indicating the living state of the bacteria as shown in Fig. 5(c). After the first injection of CM15 at the MIC and the minimal increase in surface roughness, the analyzed bacteria still appeared in the green fluorescence channel, indicating their living state (Fig.5(d)). After the injection of five times the MIC of CM15, all bacteria were only observable in the red fluorescence channel, indicating their dead state, as shown in Fig. 5(e).

After each experiment, decontamination of the whole bacterial trapping device including all microfluidic parts was performed with ethanol or isopropyl alcohol. The microfluidic chip assembly parts were additionally decontaminated using piranha solution, as described in the materials and methods section regarding the reusability of the microfluidic chip. The microfluidic chip assembly compounds were used throughout all experiments after thorough cleaning and reassembly.

Care must be taken for consecutive AFM experiments with live bacteria or long-term experiments, since bacteria can adhere to the AFM tip and cause imaging artifacts. For the next reuse, the AFM tip along with the microfluidic chip assembly parts need thorough decontamination and cleaning after an experiment. 


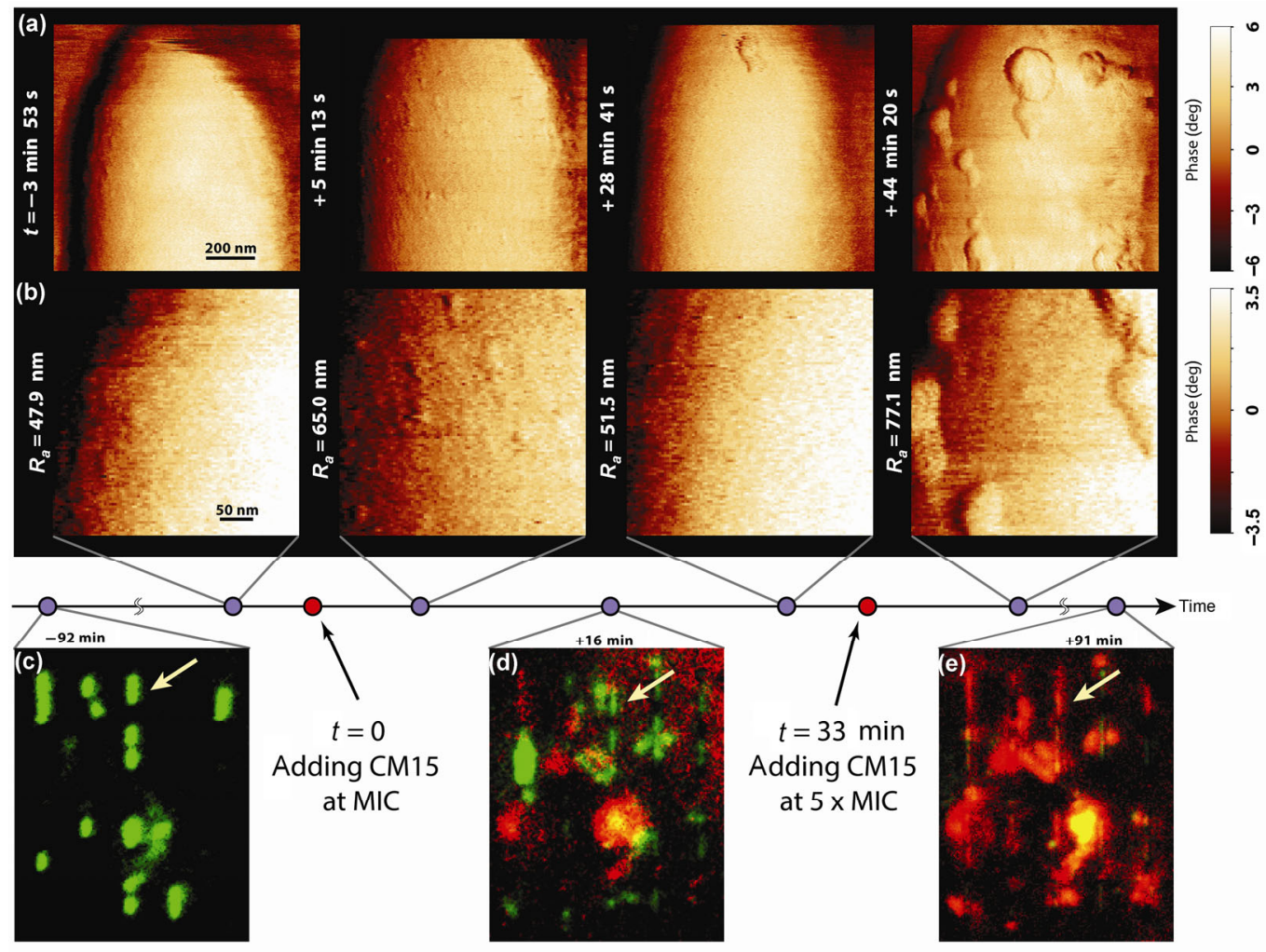

Figure 5 (a) Time sequence of the surface morphology changes of an E. coli bacterium imaged in lysogeny broth growth medium. (b) Magnified area of (a) with a higher contrast. $R_{a}$ indicates the surface roughness and is the arithmetical mean deviation of all points on the height map of the bacterial surface. The $R_{a}$ values were measured on the surface of the bacterium in the corresponding AFM height image. The antimicrobial peptide CM15 was injected at time $t=0 \mathrm{~s}$. Before the injection of CM15 at its minimum inhibitory concentration, the bacterial surface was smooth. Immediately afterwards, a faint surface roughening was observed. This roughening vanished and the surface appeared to be smooth again after $28 \mathrm{~min}$. After a consecutive injection with five times the minimum inhibitory concentration of CM15, the bacterial envelope developed visible and irreversible effects of surface damage, as shown on the rightmost subfigures. (c)-(e) Fluorescence images of trapped E. coli obtained during AFM imaging. Image (c) was obtained in the green channel, indicating the live state of bacteria stained with the LIVE/DEAD ${ }^{\circledR}$ BacLight ${ }^{\mathrm{TM}}$ stain. Images (d) and (e) show the overlay of the green and red fluorescence channels, where red indicates the dead state of bacteria. The arrow shows the targeted bacterium for AFM imaging as shown in the sequence (a). Image (c) was taken at 92 min before the injection of CM15, (d) was taken at 16 min after, and (e) was taken at 91 min after the first injection of CM15.

Over the years, various protocols for bacterial immobilization for AFM have been established [37, 38]. Specific gram-positive (S. aureus) as well as gramnegative (E. coli) bacteria showed superior attachment to gelatin-coated substrates compared to poly-Llysine-coated ones [39]. However, the same gelatin substrate coating did not allow the adequate immobilization of Synechococcus leopoliensis, a different gram-negative bacterial species [40], even though the same immobilization protocol was followed [41]. Therefore, standard immobilization protocols with chemical substrate modification often have to be modified [42] or new protocols have to be developed
[43]. The optimal coating depends strongly on the bacterial species and strain, as well as the surrounding liquid imaging medium. Nevertheless, the bacterial envelope properties are altered when the bacterium is attached to a chemically modified surface, which can additionally trigger bacterial responses and compromise the analysis of the bacterial viability $[16,17,44,45]$.

Physical immobilization leaves the bacterial envelope chemically intact and allows a reliable and robust immobilization. Proven techniques using filter pores have been further improved [46] and new physical immobilization methods have been developed. Pits 
patterned in a substrate allow bacterial immobilization after bacteria settle into the traps through the evaporation of the liquid medium [47], or bacteria need to be centrifuged into polydimethylsiloxane (PDMS) channels [48].

Our microfluidic chip assembly compounds are fabricated from chemically inert materials. In contrast, microfluidic devices made from PDMS may influence cell behavior, since uncrosslinked oligomers can interact with microorganisms, and hydrophobic molecules from the medium can be absorbed into the PDMS, which could lead to experimental artifacts [49].

Our proposed physical immobilization method is independent of the envelope composition of rodshaped bacteria, the surrounding liquid medium, and the bacterial sample preparation. AFM analysis can be conducted immediately after placing a drop of the bacterial suspension on top of the assembled microfluidic chip. This allows for versatile applications across many bacterial species and liquid media.

\section{Conclusions}

With the presented microfluidic device, the robust immobilization of bacteria is possible, accelerating the previously cumbersome sample preparation process. The fabricated bacterial traps allow the physical immobilization of motile as well as non-motile rodshaped bacteria, regardless of the liquid buffer medium. Moreover, the simultaneous monitoring of bacterial viability with an inverted fluorescence microscope allows for correlated measurements during AFM imaging.

While we demonstrated the performance of our device using rod-shaped bacteria, this trapping method would be equally applicable to round-shaped cells. In addition, the ability to reverse the trapping, eject cells, and trap new cells for analysis allows for a higher throughput of single-cell measurements.

With the present microfluidic device, we were able to observe bacterial surface phenomena that are dependent of the surrounding physiological medium. Subsequently, bacteria can be exposed to antibiotic compounds in physiological conditions, mimicking the gastrointestinal tract. We expect that additional studies using the combined AFM and optical analysis enabled by our device could yield a better understanding of the actions of antibiotics within the intestinal environment at the single-cell level.

\section{Materials and methods}

\subsection{Fabrication of the microfluidic chip assembly parts}

Silicon nitride was deposited on a $100 \mathrm{~mm}$ SOI wafer (Soitec, Bernin, France) by low-pressure chemical vapor deposition in a furnace (c.E2000, Centrotherm Photovoltaics, Blaubeuren, Germany) at temperatures ranging between 820 and $850{ }^{\circ} \mathrm{C}$. The positive e-beam resist ZEP520A (Zeon Corporation, Tokyo, Japan) was used as a mask for e-beam lithography on the top side of the SOI wafer, and a standard positive photoresist (AZ 1512, MicroChemicals, Ulm, Germany) was used for the back side patterning of silicon nitride. Silicon nitride etching was done by reactive ion etching using a $\mathrm{He}, \mathrm{H}_{2}$, and $\mathrm{C}_{4} \mathrm{~F}_{8}$-based plasma (LPX, SPTS Technologies, Newport, UK). The top side anisotropic etching was done in $40 \% \mathrm{KOH}$ solution for $3 \mathrm{~min}$ at $60{ }^{\circ} \mathrm{C}$ and the back side etching was done for $\sim 6 \mathrm{~h}$ in $23 \% \mathrm{KOH}$ solution at $90{ }^{\circ} \mathrm{C}$. Etching was stopped when the membrane containing the bacterial traps was visible, indicating a through-wafer etch. To protect the top side features of the wafer, we used a 6 - $\mu$ m-thick top side protective and supporting polymer layer (ProTEK ${ }^{\circledR}$ B3, Brewer Science), which stayed intact during the back side $\mathrm{KOH}$ etching. In addition to protecting the top silicon layer against $\mathrm{KOH}$, this polymer layer also served as a mechanical support for the finished chips so that no silicon support bridges were required. The removal of the protective polymeric layer and the etching of the silicon dioxide layer were performed in $50 \%$ hydrofluoric acid on a chip-bychip basis for 3 min with light agitation of the chip in the liquid.

The microfluidic chip holders (Fig. 1(c)) were fabricated using standard silicon microfabrication with through-wafer $\mathrm{KOH}$ etching. We applied an etchthrough with $23 \% \mathrm{KOH}$ at $90{ }^{\circ} \mathrm{C}$ to create the inlet and outlet cavities, and a central square hole with the same slope angle as the SOI chips. A water-resistant epoxy glue (2 Ton ${ }^{\circledR}$ epoxy, Devcon, Danvers, MA, 
USA) was applied around the edge of the embedded SOI chip and cured for at least $1 \mathrm{~h}$ prior to use. A 200- $\mu$ m-thick borosilicate glass slide (MEMpax ${ }^{\circledR}$, Schott, Mainz, Germany) was diced out of a $100 \mathrm{~mm}$ wafer and assembled with the silicon holder through a 100- $\mu \mathrm{m}$-thick double-sided adhesive tape containing pre-cut channels (FAD 100S, FLEXcon, Spencer, MA, USA). The channels connecting the central cavity with the connection ports were fabricated using a cutting plotter (Craft ROBO Pro, Graphtec, Tokyo, Japan).

The microfluidic chip assembly is clamped in a custom-built aluminum mount that interfaces the chip with a pressure controller (AF1 Dual, Elveflow, Paris, France). The aluminum mount together with the microfluidic chip assembly were designed to work at room temperature. The whole assembly was placed in a homebuilt combined AFM/optical microscope [22].

\subsection{Fluid flow analysis}

Finite element analysis of the fluid flow through the bacterial traps was performed in COMSOL Multiphysics $^{\circledR} 4.4$ (COMSOL, Burlington, MA, USA). The interface between the solid (silicon) and the liquid (water) parts of the model was defined through a no-slip boundary condition. At the opening below the membrane, defined as the outlet, an initial pressure of -20 mbar was set, as used during the experiments. The liquid above the membrane had open boundary conditions, whereas the liquid below the membrane was confined through the silicon walls of the 3D model. For the volume flow analysis, we integrated the velocity field in the direction of fluid motion over the surface of the traps from the COMSOL Multiphysics ${ }^{\circledR}$ program, taking into account the fluid flow as function of the no-slip boundary condition.

The force approximation is based on a bacterium modeled as a cylinder with a diameter $D$ and a length $L$. The force applied on an infinitesimal surface is $\delta \boldsymbol{F}=\Delta P \cdot \mathrm{d} A \cdot \boldsymbol{n}$, where $\Delta P$ is the difference of pressure between the two sides of the surface, $\mathrm{d} A$ is an infinitesimal surface, and $n$ is a unit vector normal to the surface. After integration on the whole surface of the bacterium we derive Eq. (1)

$$
\boldsymbol{F}=\iint_{\Sigma} \Delta P \cdot \boldsymbol{n} \cdot \mathrm{d} A=\left(P_{1}-P_{2}\right) D \cdot L \cdot \boldsymbol{u}_{z}
$$

where $\Sigma$ is the bacterial surface, $P_{1}$ is the pressure underneath the traps, $P_{2}$ atmospheric pressure, and $\boldsymbol{u}_{z}$ a vertical unit vector. For a bacterium with $D=$ $900 \mathrm{~nm}, L=3.5 \mu \mathrm{m}$, and an applied pressure of $\left|P_{1}-P_{2}\right|=20$ mbar, we obtain a force $F=6.3 \mathrm{nN}$ that is acting on the bacterium. It is important to note that this calculation is only intended as an approximation, to show the order of magnitude of the resulting forces on a trapped bacterium. The actual forces acting on the bacteria can deviate significantly, for example, when the area of the bacterium that is exposed to the bottom part of the traps is smaller or when the bacterium only partially populates the trap, allowing fluid to pass through.

\subsection{Bacterial preparation}

E. coli bacteria (strains DH5 $\alpha$, BL21, and K-12) and B. subtilis (strain PY79) were grown overnight from a single colony in LB growth medium at $37^{\circ} \mathrm{C}$. Then, $10 \mu \mathrm{L}$ of the cell suspension was regrown in fresh LB medium for $3 \mathrm{~h}$, and $1 \mathrm{~mL}$ of the regrown solution was diluted 10,000 times prior to use in subsequent experiments. For Fig. 4(c), $1 \mathrm{~mL}$ of the regrown bacterial suspension was centrifuged for $1 \mathrm{~min}$ at $2 \times g$ and washed with $1 \mathrm{~mL}$ LB medium. The centrifugation and washing steps were repeated 3 times. The solution was diluted 1:100 in LB medium.

\subsection{Reusability of the microfluidic chip}

The microfluidic chip holder and the nanofluidic traps can be disassembled and reused. After each experiment, we soaked the microfluidic chip assembly in acetone overnight to dissolve the double-sided sticky tape and detach the borosilicate glass slide from the silicon holder. Moreover, the acetone weakened the bond between the silicon holder and the epoxy glue around the microfluidic SOI chip. All parts were thoroughly rinsed with Milli- ${ }^{\circledR}$ water and cleaned using piranha solution $\left(\mathrm{H}_{2} \mathrm{SO}_{4}: \mathrm{H}_{2} \mathrm{O}_{2}\right.$ in a volume ratio of 3:1) at $100{ }^{\circ} \mathrm{C}$ for $15 \mathrm{~min}$. Then, all parts were rinsed twice in Milli- $Q^{\circledR}$ water and blow-dried with nitrogen before reassembly. We optimized the bacterial traps design together with the microfluidic chip assembly and cleaning techniques for the immobilization of bacteria during every experiment. 


\subsection{Experimental setup}

Prior to an experiment, the channel of the microfluidic chip assembly was flushed with Milli- ${ }^{\circledR}$ water or LB medium. During this step, special care was taken to remove any air bubbles in the fluidic chamber beneath the membrane, allowing an undisturbed optical observation with the inverted microscope. The aluminum mount was placed into a custom-built frame, between an inverted optical microscope (IX73, Olympus, Tokyo, Japan) and an AFM head (Dimension FastScan, Bruker, Billerica, MA, USA) on a vibration isolation table. For optical microscopy we used a $60 \times$ air microscope objective (LUCPLFLN, Olympus). The custom-built frame is described in a previous work [22]. The pressure difference across the bacterial traps (Fig. 1(d)) was created by clogging the outlet and controlling the pressure on the inlet with a pressure controller (AF1 Dual, Elveflow). The prepared bacteria solution was added on top of the microfluidic chip assembly. After the trapping of bacteria was observed using the fluorescence microscope, the top side of the chip was rinsed with the identical medium as that used for the bacterial solution, using pipettes to remove bacteria that were not physically immobilized. Except for the bacterial solution, all liquids were filtered through a $200 \mathrm{~nm}$ Millipore ${ }^{\mathrm{TM}}$ filter before being inserted into the microfluidic channel or onto the top side of the microfluidic chip assembly. The liquid in the channel was LB medium or Milli- $Q^{\circledR}$ water, corresponding to the buffer medium in which the bacteria were analyzed.

We also used a second custom-built setup consisting of a Dimension Icon AFM head (Bruker) and an inverted optical microscope (IX81, Olympus) on top of a vibration isolation table (AVI-200-XL, Table Stable, Mettmenstetten, Switzerland). The fluorescence excitation originated from a mercury arc lamp (X-Cite ${ }^{\circledR} 120$, Excelitas Technologies, Wiesbaden, Germany). Images were recorded with an iXon3 (Andor, Belfast, UK) camera. For AFM imaging, a Nanoscope V controller in tapping and PeakForce $\mathrm{QNM}^{\circledR}$ modes was used.

\subsection{AFM and fluorescence microscopy imaging}

The immobilized bacterium in Fig. 4(a) was imaged in LB growth medium in tapping mode using a ScanAsyst ${ }^{\circledR}$-Fluid (Bruker) cantilever. After initial trapping, the applied pressure was released and the bacterium remained physically immobilized. The bacterium shown in Fig. 4(b) was imaged in PeakForce $\mathrm{QNM}^{\circledR}$ mode using a ScanAsyst ${ }^{\circledR}$-Fluid cantilever with a $1.6 \mathrm{~N} \cdot \mathrm{m}^{-1}$ measured spring constant. During the imaging procedure, the pressure in the microfluidic chamber underneath the membrane was kept between 20 and 100 mbar below atmospheric pressure. Bacteria were imaged in Milli- $Q^{\circledR}$ water at room temperature and we did not observe any growth of the bacterium. The imaging in Fig. 4(c) was performed with 10 lines $\cdot \mathrm{s}^{-1}$ in tapping mode with a FastScan-DX probe (Bruker) at a measured resonance frequency of $\sim 100 \mathrm{kHz}$. We used a pressure difference in the range of -50 to -150 mbar to guide bacteria towards the traps, and a positive pressure difference in the same range to release bacteria from the traps. The trapping and releasing of individual cells happens within the acquisition of an AFM image and the images depicted in Fig. 4(c) show the steps of completed trapping or releasing. The bacterium depicted in Fig. 5(a) was imaged in tapping mode using a Hydra-All-B (Applied Nanostructures, Mountain View, CA, USA) cantilever in LB growth medium.

For the fluorescence microscopy, bacteria were stained with the LIVE/DEAD ${ }^{\circledR}$ BacLight $^{\mathrm{TM}}$ bacterial viability kit by adding $1.5 \mu \mathrm{L}$ of SYTO9 and $1.5 \mu \mathrm{L}$ of propidium iodide into $1 \mathrm{~mL}$ of diluted bacterial solution, followed by incubation for $15 \mathrm{~min}$ in the absence of light. Fluorescence calibration tests were conducted on the used equipment with bacteria immobilized on poly-L-lysine-coated glass cover slips, as described in previous work [8], to characterize the bacterial live and dead fluorescence signals of our system according to recent findings [50].

The correlated fluorescence imaging and AFM scanning in tapping mode were performed while the AFM cantilever tip was engaged. The fluorescence image was taken at the end of an AFM frame and we manually triggered the fluorescence laser excitation during the off-state of the AFM laser. The AFM laser was switched off through a custom LabVIEW (National Instruments, Austin, TX, USA) script while in ramp 
mode to take the fluorescence image. The fluorescence figures were processed using ImageJ [51, 52]. The color was added in Image J to represent the respective fluorescence channel. All fluorescence images are normalized in respect to the background.

For the experiments in the PeakForce $\mathrm{QNM}^{\circledR}$ mode, we calibrated the AFM cantilever next to the membrane in liquid using the thermal tune method [53]. AFM data in 2D were processed with Gwyddion [54] using standard modification commands and the 3D AFM image in Fig. 4(b) was created using NanoScope Analysis 1.7 software (Bruker).

\section{Acknowledgements}

This work was funded by the Swiss National Science Foundation (Nos.205321_134786, 205320_152675), and by the European Union FP7/2007-2013/ERC under Grant Agreement No. 307338-NaMic, and Eurostars E!8213.

Electronic Supplementary Material: Supplementary material (additional AFM figures of B. subtilis and $E$. coli $)$ is available in the online version of this article at https://doi.org/10.1007/s12274-017-1604-5.

\section{References}

[1] Beveridge, T. J.; Graham, L. L. Surface layers of bacteria. Microbiol. Rev. 1992, 55, 684-705.

[2] Butt, H. J.; Wolff, E. K.; Gould, S. A. C.; Dixon Northern, B.; Peterson, C. M.; Hansma, P. K. Imaging cells with the atomic force microscope. J. Struct. Biol. 1990, 105, 54-61.

[3] Pum, D.; Sleytr, U. B. Monomolecular reassembly of a crystalline bacterial cell surface layer (S-layer) on untreated and modified silicon surfaces. Supramol. Sci. 1995, 2, 193-197.

[4] Schabert, F.; Henn, C.; Engel, A. Native Escherichia coli OmpF porin surfaces probed by atomic force microscopy. Science 1995, 268, 92-94.

[5] Drake, B.; Prater, C.; Weisenhorn, A.; Gould, S.; Albrecht, T.; Quate, C.; Cannell, D.; Hansma, H.; Hansma, P. Imaging crystals, polymers, and processes in water with the atomic force microscope. Science 1989, 243, 1586-1589.

[6] Beaussart, A.; Baker, A. E.; Kuchma, S. L.; El-Kirat-Chatel, S.; O'Toole, G. A.; Dufrêne, Y. F. Nanoscale adhesion forces of Pseudomonas aeruginosatype IV pili. ACS Nano 2014, 8, 10723-10733.

[7] Hayhurst, E. J.; Kailas, L.; Hobbs, J. K.; Foster, S. J. Cell wall peptidoglycan architecture in Bacillus subtilis. Proc. Natl. Acad. Sci. USA 2008, 105, 14603-14608.

[8] Fantner, G. E.; Barbero, R. J.; Gray, D. S.; Belcher, A. M. Kinetics of antimicrobial peptide activity measured on individual bacterial cells using high-speed atomic force microscopy. Nat. Nanotechnol. 2010, 5, 280-285.

[9] Dufrêne, Y. F.; Martínez-Martín, D.; Medalsy, I.; Alsteens, D.; Müller, D. J. Multiparametric imaging of biological systems by force-distance curve-based AFM. Nat. Methods 2013, 10, 847-854.

[10] Ando, T.; Uchihashi, T.; Scheuring, S. Filming biomolecular processes by high-speed atomic force microscopy. Chem. Rev. 2014, 114, 3120-3188.

[11] Radmacher, M.; Tillmann, R. W.; Fritz, M.; Gaub, H. E. From molecules to cells: Imaging soft samples with the atomic force microscope. Science 1992, 257, 1900-1905.

[12] Dufrêne, Y. F. Using nanotechniques to explore microbial surfaces. Nat. Rev. Microbiol. 2004, 2, 451-460.

[13] Karrasch, S.; Dolder, M.; Schabert, F.; Ramsden, J.; Engel, A. Covalent binding of biological samples to solid supports for scanning probe microscopy in buffer solution. Biophys. J. 1993, 65, 2437-2446.

[14] Kasas, S.; Ikai, A. A method for anchoring round shaped cells for atomic force microscope imaging. Biophys. J. 1995, $68,1678-1680$.

[15] Razatos, A.; Ong, Y. L.; Sharma, M. M.; Georgiou, G. Molecular determinants of bacterial adhesion monitored by atomic force microscopy. Proc. Natl. Acad. Sci. USA 1998, 95, 11059-11064.

[16] Meyer, R. L.; Zhou, X. F.; Tang, L.; Arpanaei, A.; Kingshott, P.; Besenbacher, F. Immobilisation of living bacteria for AFM imaging under physiological conditions. Ultramicroscopy 2010, 110, 1349-1357.

[17] Liu, Y.; Strauss, J.; Camesano, T. A. Adhesion forces between Staphylococcus epidermidis and surfaces bearing self-assembled monolayers in the presence of model proteins. Biomaterials 2008, 29, 4374-4382.

[18] Vadillo-Rodríguez, V.; Busscher, H. J.; Van Der Mei, H. C.; De Vries, J.; Norde, W. Role of lactobacillus cell surface hydrophobicity as probed by AFM in adhesion to surfaces at low and high ionic strength. Colloids Surf. B Biointerfaces 2005, 41, 33-41.

[19] Zhang, T.; Chao, Y. Q.; Shih, K.; Li, X. Y.; Fang, H. H. P. Quantification of the lateral detachment force for bacterial cells using atomic force microscope and centrifugation. Ultramicroscopy 2011, 111, 131-139. 
[20] Vadillo-Rodriguez, V.; Busscher, H. J.; Norde, W.; de Vries, J.; Dijkstra, R. J. B.; Stokroos, I.; van der Mei, H. C. Comparison of atomic force microscopy interaction forces between bacteria and silicon nitride substrata for three commonly used immobilization methods. Appl. Environ. Microbiol. 2004, 70, 5441-5446.

[21] van der Mei, H. C.; Busscher, H. J.; Bos, R.; de Vries, J.; Boonaert, C. J. P.; Dufrêne, Y. F. Direct probing by atomic force microscopy of the cell surface softness of a fibrillated and nonfibrillated oral streptococcal strain. Biophys. J. 2000, 78, 2668-2674.

[22] Odermatt, P. D.; Shivanandan, A.; Deschout, H.; Jankele, R.; Nievergelt, A. P.; Feletti, L.; Davidson, M. W.; Radenovic, A.; Fantner, G. E. High-resolution correlative microscopy: Bridging the gap between single molecule localization microscopy and atomic force microscopy. Nano Lett. 2015, 15, 4896-4904.

[23] Hertz, H. Ueber die Berührung fester elastischer Körper. $J$. Für Die Reine Und Angew. Math. 1882, 92, 156-171.

[24] Johnson, K. L.; Kendall, K.; Roberts, A. D. Surface energy and the contact of elastic solids. Proc. Roy. Soc. A: Math. Phys. Eng. Sci. 1971, 324, 301-313.

[25] Derjaguin, B. V; Muller, V. M.; Toporov, Y. P. Effect of contact deformations on the adhesion of particles. J. Colloid Interface Sci. 1975, 53, 314-326.

[26] Butt, H. J.; Cappella, B.; Kappl, M. Force measurements with the atomic force microscope: Technique, interpretation and applications. Surf. Sci. Rep. 2005, 59, 1-152.

[27] Huang, L. R.; Cox, E. C.; Austin, R. H.; Sturm, J. C. Continuous particle separation through deterministic lateral displacement. Science 2004, 304, 987-990.

[28] Wyatt Shields IV, C.; Reyes, C. D.; López, G. P. Microfluidic cell sorting: Areview of the advances in the separation of cells from debulking to rare cell isolation. Lab Chip 2015, 15, 1230-1249.

[29] Dufrêne, Y. F. Atomic force microscopy in microbiology: New structural and functional insights into the microbial cell surface. mBio 2014, 5, e01363-14.

[30] Longo, G.; Kasas, S. Effects of antibacterial agents and drugs monitored by atomic force microscopy. Wiley Interdiscip. Rev. Nanomed. Nanobiotechnol. 2014, 6, 230-244.

[31] Braga, P. C.; Ricci, D. Atomic force microscopy: Application to investigation of Escherichia colimorphology before and after exposure to cefodizime. Antimicrob. Agents Chemother. 1998, 42, 18-22.

[32] Francius, G.; Domenech, O.; Mingeot-Leclercq, M. P.; Dufrêne, Y. F. Direct observation of Staphylococcus aureus cell wall digestion by lysostaphin. J. Bacteriol. 2008, 190, 7904-7909.
[33] Yeaman, M. R.; Yount, N. Y. Mechanisms of antimicrobial peptide action and resistance. Pharmacol. Rev. 2003, 55, 27-55.

[34] Brogden, K. A. Antimicrobial peptides: Pore formers or metabolic inhibitors in bacteria? Nat. Rev. Microbiol. 2005, 3, 238-250.

[35] Peraro, M. D.; van der Goot, F. G. Pore-forming toxins: Ancient, but never really out of fashion. Nat. Rev. Microbiol. 2016, 14, 77-92.

[36] Sato, H.; Feix, J. B. Osmoprotection of bacterial cells from toxicity caused by antimicrobial hybrid peptide CM15. Biochemistry 2006, 45, 9997-10007.

[37] Dufrêne, Y. F. Atomic force microscopy and chemical force microscopy of microbial cells. Nat. Protoc. 2008, 3, 11321138.

[38] Liu, Y. T. ; Camesano, T. A. Immobilizing bacteria for atomic force microscopy imaging or force measurements in liquids.In Microbial Surfaces: Structure, Interactions, and Reactivity. Camesano, T. A.; Mello, C. M., Eds.; American Chemical Society: San Francisco, 2008; pp. 163-188.

[39] Doktycz, M. J.; Sullivan, C. J.; Hoyt, P. R.; Pelletier, D. A.; $\mathrm{Wu}, \mathrm{S}$.; Allison, D. P. AFM imaging of bacteria in liquid media immobilized on gelatin coated mica surfaces. Ultramicroscopy 2003, 97, 209-216.

[40] Obst, M.; Dittrich, M. Living under an atomic force microscope. Geobiology 2005, 3, 179-193.

[41] Allison, D. P.; Sullivan, C. J.; Mortensen, N. P.; Retterer, S. T.; Doktycz, M. Bacterial immobilization for imaging by atomic force microscopy. J. Vis. Exp. 2011, 2880.

[42] Pelling, A. E.; Li, Y.; Shi, W.; Gimzewski, J. K. Nanoscale visualization and characterization of Myxococcus xanthus cells with atomic force microscopy. Proc. Natl. Acad. Sci. USA 2005, 102, 6484-6489.

[43] Read, N.; Connell, S.; Adams, D. G. Nanoscale visualization of a fibrillar array in the cell wall of filamentous cyanobacteria and its implications for gliding motility. J. Bacteriol. 2007, 189, 7361-7366.

[44] Liu, B. Y.; Zhang, G. M.; Li, X. L.; Chen, H. Effect of glutaraldehyde fixation on bacterial cells observed by atomic force microscopy. Scanning 2012, 34, 6-11.

[45] Colville, K.; Tompkins, N.; Rutenberg, A. D.; Jericho, M. H. Effects of poly(L-lysine) substrates on attached Escherichia coli bacteria. Langmuir 2010, 26, 2639-2644.

[46] Turner, R. D.; Thomson, N. H.; Kirkham, J.; Devine, D. Improvement of the pore trapping method to immobilize vital coccoid bacteria for high-resolution AFM: A study of Staphylococcus aureus. J. Microsc. 2010, 238, 102-110.

[47] Kailas, L.; Ratcliffe, E. C.; Hayhurst, E. J.; Walker, M. G.; Foster, S. J.; Hobbs, J. K. Immobilizing live bacteria for 
AFM imaging of cellular processes. Ultramicroscopy 2009, 109, 775-780.

[48] Chen, P. P.; Xu, L. P.; Liu, J.; Hol, F. J. H.; Keymer, J. E.; Taddei, F.; Han, D.; Lindner, A. B. Nanoscale probing the kinetics of oriented bacterial cell growth using atomic force microscopy. Small 2014, 10, 3018-3025.

[49] Regehr, K. J.; Domenech, M.; Koepsel, J. T.; Carver, K. C.; Ellison-Zelski, S. J.; Murphy, W. L.; Schuler, L. A.; Alarid, E. T.; Beebe, D. J. Biological implications of polydimethylsiloxane-based microfluidic cell culture. $L a b$ Chip 2009, 9, 2132-2139.

[50] Stiefel, P.; Schmidt-Emrich, S.; Maniura-Weber, K.; Ren, Q. Critical aspects of using bacterial cell viability assays with the fluorophores SYTO9 and propidium iodide. BMC Microbiol. 2015, 15, 36.

[51] Schindelin, J.; Arganda-Carreras, I.; Frise, E.; Kaynig, V.; Longair, M.; Pietzsch, T.; Preibisch, S.; Rueden, C.; Saalfeld, S.; Schmid, B.et al. Fiji: An open-source platform for biological-image analysis. Nat. Methods 2012, 9, 676-682.

[52] Schneider, C. A.; Rasband, W. S.; Eliceiri, K. W. NIH Image to ImageJ: 25 years of image analysis. Nat. Methods 2012, 9, 671-675.

[53] Hutter, J. L.; Bechhoefer, J. Calibration of atomic-force microscope tips. Rev. Sci. Instrum. 1993, 64, 1868-1873.

[54] Nečas, D.; Klapetek, P. Gwyddion: An open-source software for SPM data analysis. Cent. Eur. J. Phys. 2012, 10, 181-188. 